\title{
Le « savoir-vivre » épistolaire
}

Höflichkeit und Briefkultur

Epistolary Etiquette

\section{Alain Montandon}

\section{OpenEdition}

\section{Journals}

Édition électronique

URL : http://journals.openedition.org/ceg/843

DOI : $10.4000 /$ ceg. 843

ISSN : 2605-8359

\section{Éditeur}

Presses Universitaires de Provence

Édition imprimée

Date de publication : 30 mai 2016

Pagination : 35-46

ISBN : 979-10-320-0067-0

ISSN : 0751-4239

\section{Référence électronique}

Alain Montandon, « Le « savoir-vivre » épistolaire », Cahiers d'Études Germaniques [En ligne], 70 | 2016,

mis en ligne le 17 décembre 2017, consulté le 25 novembre 2020. URL : http://

journals.openedition.org/ceg/843; DOI : https://doi.org/10.4000/ceg.843 


\title{
Le " savoir-vivre " épistolaire ${ }^{1}$
}

\author{
Alain MONTANDON \\ Université Blaise-Pascal (Clermont II)
}

Lorsqu'on pose la question de l'adresse de la lettre, on est frappé par l'extraordinaire diversité des destinataires du genre épistolaire, au point même que la notion générique fait problème. Ainsi pour Gustave Lanson, il n’y aurait pas plus de genre épistolaire, que de genre oral. Et il est vrai que, outre le cas particulier du roman par lettres, la littérature épistolaire offre un domaine d'une extraordinaire variété. Qu'y aurait-il de commun entre une lettre d'affaire, une lettre d'amour, une lettre diplomatique, une lettre de réclamation, un billet, une lettre de civilité, un billet de condoléances, de félicitations, de vœux, une lettre officielle ou une lettre familière, une lettre de créance ou une lettre de démission, une lettre polémique et pamphlétaire, une lettre curieuse et exotique comme les Lettres persanes de Montesquieu, les lettres de confession telles que Rousseau en rédigea ou encore les lettres didactiques, morales, démonstratives ? Cicéron, qui fut un modèle majeur de l'art épistolaire, offrait déjà une large gamme de tours et de tons dans son abondante production, comme le note Arbi Dhifaoui dans son cours sur la littérature épistolaire du XVIII ${ }^{\mathrm{e}}$ siècle : sociabilité amicale mêlée aux questions politiques et morales dans ses lettres Ad Atticus, questions privées dans les lettres Ad Familiares, conseils de morale dans celles qui sont adressées à son frère cadet Quintius. La Chétardie distinguait quant à lui cinq genres de lettres :

Il y a cinq sortes de genres d'écrire, les Lettres d'affaires, les Lettres familières, les Lettres galantes, les Billets doux, et les Lettres de compliment. Quand on écrit pour affaire, il faut entrer d'abord dans le sujet, se servir de termes propres à la chose dont on parle, s'exprimer clairement et sans confusion. [...] Les Lettres familières portent leur titre avec elles, elles doivent être toutes unies. Il faut dès lors que votre Ami les reçoit, il s'imagine que vous lui parlez, qu'il puisse dire, je le reconnais, il me semble que je l'entends, voilà son caractère. Cependant quoique l'esprit n'y doive pas briller, il faut pourtant que les termes soient justes, et qu'on y remarque un certain tour de qualité, où les personnes du commun ne parviennent que rarement. Les Lettres galantes ont un caractère particulier. Il faut qu'il y entre de l'enjouement et du badinage dans les endroits qui en sont susceptibles; que le style en soit doux et coulant ; la louange délicate et fine ; la raillerie flatteuse et insinuante ; que les périodes soient courtes, parce que plus il y en a dans une lettre, plus il y a de pensées, qu'il n'y ait rien de languissant ni de froid ; et comme ces lettres-là ne sont faites que pour plaire, il faut prendre garde qu'elles n'ennuient. Pour les Billets doux, comme c'est l'ouvrage du cœur, il faut qu'il y paraisse plus de tendresse que d'esprit. [...] Les Lettres de compliment

1. Une partie de cette contribution est la version complétée d'un article paru dans le numéro 7 (" Laiškas literatūroje ir kultūroje/The letter in literature and culture ", 2015) de la revue Acta litteraria comparativa. 
ont pour objets certains devoirs que la société civile oblige de se rendre les uns aux autres dans les différentes occasions qui se présentent. [...] Ces lettres là doivent être civiles et obligeantes et n'être guère plus longues que les compliments qu'on se fait à la rencontre².

Furetière, dans son Dictionnaire, notait en 1690 cette grande variété quand il écrivait de la lettre :

Se dit d'un écrit qu'on envoie à un absent pour lui faire entendre sa pensée. Les amis s'écrivent des lettres de compliments, de nouvelles, de sciences, de curiosités, de consolation ; les amants des lettres de galanterie, de tendresse; les procureurs, les agents, des lettres d'affaires, de recommandations ; les banquiers des lettres de change, de créance, d'avis ${ }^{3}$...

L'élément commun réside dans la représentation sociale et psychologique du destinataire, et les différentes représentations des destinataires déterminent à la fois le contenu et la forme de la missive. Aussi, la civilité épistolaire repose d'abord sur l'identité du destinataire et sa représentation. Et cela implique une diversité de style que notait déjà Érasme quand il écrivait dans De conscribendis epistolis : "Poser, dans les lettres, une manière unique comme exigence ou comme principe - attitude que l'on constate parfois chez les esprits les plus cultivés -, c'est vraiment à mes yeux adopter, en une matière si complexe et dont la variété tend à l'infini, une position étroite et sévère à l'excès ${ }^{4}$ ". Cela sera repris par la suite par quantité de gens, s'opposant en cela à certains traités épistolaires assez rigides.

La lettre est, on le sait, destinée à remédier à une absence, au défaut de la présence de l'autre, et cela pour s'en rapprocher, pour donner une information, formuler une demande ou maintenir une communication. "Qu'est-ce qu'une lettre? Un écrit envoyé à une personne absente pour lui faire savoir ce que nous lui dirions si nous étions en état de lui parler " disait Vaumorière en $1689^{5}$. C'est dire qu'elle est le substitut d'un entretien oral auquel sa nature écrite ajoute parfois un poids supplémentaire. Ce que le Chevalier de Méré exprime de manière sagace :

On écrit des choses qu'on ne prononce jamais, et qui ne sont faites que pour être lues [...] et parce que les lettres ne se prononcent point ; car encore qu'on en lise tout haut, ce n'est pas ce qu'on appelle prononcer, on ne les doit pas écrire tout-à-fait comme on parle. Pour preuve de cela, qui verrait une personne à qui l'on vient d'écrire une lettre, quoi qu'elle fust excellente, on ne luy diroit pas les mesmes choses qu'on luy écrivoit, ou pour le moins, on ne les luy diroit pas de la mesme façon. Il est pourtant bon lorsquon écrit de s'imaginer en quelque sorte qu'on parle, pour ne rien mettre qui ne soit naturel, et qu'on pût dire dans le monde 6 .

2. Trotti de La Chétardie, Instructions pour un jeune seigneur, Paris, Nicolas Le Gras, 1702 [1 $1^{\text {re }}$ éd. 1683], Première partie, p. 142-143.

3. Furetière, Dictionnaire universel, contenant généralement tous les mots françois [...], La Haye / Rotterdam, Arnout \& Reinier, 1670, t. 2, "Lettre ", p. 442.

4. "Qui in epistolis unum aliquem characterum vel requirunt, vel praescribunt, id quod ab eruditioribus etiam quibusdam video factum, ii mihi de re tam multiplici, propeque in infinitum varia, nmis auguste atque arcte videntur agere. "(Érasme, De conscribendis epistolis, Amsterdam, Boom, 1682, fo 9.)

5. Pierre Ortigue de Vaumorière, L'art de plaire dans la conversation, Paris, Jean Guignard, 1689.

6. Chevalier de Méré, Les Conversations, Paris, Claude Barbin, 1670 [1 ${ }^{\text {re }}$ éd. 1669], p. 87-88. 
Ces idées ont été textuellement reprises en Allemagne de manière récurrente dans les secrétaires (Briefsteller), qui sont des livres précisant les règles d'écriture épistolaire et offrant de nombreux modèles. Ainsi Stockhausen dans ses Grundsätze wohleingerichteter Briefe précise-t-il le statut de la lettre et bien entendu les conséquences à en tirer quant au comportement envers le destinataire : "Les lettres sont des dialogues que nous transposons par écrit et tenons avec des personnes absentes à certaines occasions ou pour certains motifs ${ }^{7}$. "

Et parce que communication à distance, la lettre affiche plus de contraintes conventionnelles: en-têtes, indications de lieu, moment et circonstances, formules introductives et formules finales (couramment dites "de politesse "), signature, et même parfois jeu du post-scriptum et des documents joints, tout cela codifie fortement le discours dans sa matérialité même ${ }^{8}$.

Ces contraintes et codes dépendent des personnes auxquelles on s'adresse et de ce qu'elles représentent. Courtin y revient dans son Nouveau traité de la civilité qui se pratique en France parmi les honnêtes gens (1671) en consacrant à cet aspect un chapitre intitulé "Qu'il faut dans une lettre considérer le style, la matière et la personne ". Les nombreux traités d'art épistolaire qui ont été édités en Italie au XVI ${ }^{\mathrm{e}}$ siècle ${ }^{9}$ et largement diffusés en France insistaient déjà sur le statut des destinataires pour prodiguer des conseils et recommandations adaptés. Les secrétaires étaient des ouvrages contenant " plusieurs modèles de lettres et de compliments pour ceux qui ne savent pas en faire ${ }^{10}$ " et qui, précisant les règles d'écriture d'une lettre, vont s'étendre à toute l'Europe de 1530 à 1660. Les règles sont essentiellement celles du decorum et des bienséances à respecter. L'âge, le sexe, la position sociale, la hiérarchie et la division des états, des conditions de la fortune dans l'Ancien Régime, etc., font l'objet des prescriptions précises en rapport avec les représentations culturelles et sociales de l'époque.

Grimarest en 1709 défınissait ainsi le cérémonial épistolaire comme celui " des égards de civilité, d'honnêteté, de respect, marqués par de certaines expressions et en certains endroits de la lettre, égards différents selon le rang, la naissance, le mérite ${ }^{11}$ ». Il distinguait deux grandes sortes de lettres : lettres en cérémonie, lettres familières. Cette distinction fait référence à la règle des trois sortes de personnes (supérieurs, égaux, inférieurs) qui gère les rapports entre les hommes dans la société d'Ancien Régime, et donc à la bienséance,

7. Johann Christoph Stockhausen, Grundsätze wohleingerichteter Briefe, Helmstedt, Weygand, 1751, p. 1: „Briefe sind schriftliche Unterredungen, die wir mit abwesenden Personen in gewissen Angelegenheiten oder Absichten anstellen."

8. Voir Alain Montandon (dir.), Dictionnaire raisonné de la politesse et du savoir-vivre, Paris, Seuil, 1995, et Marie-Claire Grassi, L'Épistolaire, Paris, Dunod, 1998.

9. Voir Jeannine Basso, Le genre épistolaire en langue italienne (1538-1662). Répertoire chronologique et analytique, Roma, Bulzoni editore/Presses Universitaires de Nancy, 1990, 2 vol.

10. Furetière, Dictionnaire, t. 3, "Secrétaire ", p. 509.

11. Jean Léonor Le Gallois, sieur de Grimarest, Traité sur la manière d'écrire des lettres et sur le cérémonial avec un discours sur ce qu'on appelle usage dans la langue française, Paris, Estienne, 1709. 
"juste rapport qui doit toujours se rencontrer entre nos âges et nos actions ${ }^{12}$ ". Les lettres familières, écrites entre égaux, suivent, elles aussi, un cérémonial car " la preuve la plus sensible de la bonne éducation est la conduite que l'on tient envers les égaux ${ }^{13}$ ".

Les secrétaires italiens au XVI ${ }^{\mathrm{e}}$ siècle avaient donné une liste de termes précis qu'il faut utiliser pour s'adresser à autrui dans les trois cas (supérieurs, égaux, inférieurs), et avaient défini leur place dans la proxémique épistolaire. Tous les théoriciens français reprennent ces règles fondamentales et les adaptent. Ainsi Grimarest distingue, en début de lettre, les règles de la suscription, titre de la personne et organisation de l'espace entre ce titre et le texte, et celles de la souscription, art de prendre congé, liaison et formule de politesse avec ou sans décalage graphique. Courtin précise lui aussi les règles de la distance inspirées par le respect: "On laisse beaucoup de blanc avant que d'écrire le corps de la lettre, différemment pourtant selon la qualité des personnes et plutôt plus que moins ${ }^{14}$ ", ce que continuera de faire au XVIII ${ }^{\mathrm{e}}$ siècle Philipon de la Madelaine qui déclare en 1761 suivre l'usage le plus autorisé en la matière :

La date se place indifféremment en haut ou au bas de la lettre. On dit la seconde plus polie, je trouve la première plus commode. Vers le quart en haut, écrire la qualification de la personne selon son rang et son état, Monsieur, Monseigneur, Mademoiselle. Entre la qualification et le début de lettre, laissez un intervalle plus ou moins grand selon le respect que vous lui devez, c'est ce qu'on appelle communément donner la ligne. Il n'est plus guère d'usage de donner la ligne, sauf aux femmes et aux supérieurs. Quand on ne donne pas la ligne, il faut placer Monsieur le plus tôt qu'il se peut ${ }^{15}$.

L'ampleur du décalage graphique souligne toujours la déférence. Respect, soumission et humilité sont dus à un supérieur. La formule envers le Roi est donc "Très humble, très obéissant, très fidèle sujet et serviteur " avec grand décalage graphique, marque de l'extrême distance sociale donc d'extrême respect.

La qualité du papier importe également comme signe de bienséance et de politesse. Courtin remarque que " pour la cérémonie de l'écriture, d'inférieur à supérieur, qu'il est plus respectueux de se servir de grand papier que de petit et que le papier sur lequel on écrit doit être double et non en simple demi-feuille, quand on n'écrirait à la première page que six lignes ${ }^{16}$ ". De même il était de bon ton jusqu'en 1850, en dépit de l'arrivée sur le marché des enveloppes gommées, de faire soi-même ses enveloppes et d'avoir toujours avec soi une honnête écritoire qui contient l'important attirail d'écriture, notamment les types de papiers ainsi qu'un tiroir pour ranger les lettres reçues, lettres qu'il est préférable

12. Cf. Marie-Claire Grassi, "L'art épistolaire français (XVIII et XIX siècles) ", in Montandon (éd.), Pour une histoire des traités de savoir-vivre en Europe, Clermont-Ferrand, Publications de la Faculté des Lettres, 1994, p. 307-336, ici p. 308.

13. Antoine de Courtin, Nouveau Traité de la civilité qui se pratique en France parmi les honnestes gens, Paris, Hélie Josset, 1677 [1 ${ }^{\text {re }}$ éd. 1671], p. 222 (rééd. par M.C. Grassi, Saint-Étienne, Presses Universitaires, 1998).

14. Courtin, ibid., p. 142.

15. Louis Philipon de la Madelaine, Modèles de lettres sur différents sujets, Lyon, Bruyset-Ponthus, 1761, p. 60.

16. Courtin, Traité, p. 152. 
de numéroter. Il convient de remarquer que les usages changent en même temps que les interactions sociales et qu'au cours du XIX ${ }^{\mathrm{e}}$ siècle le cérémonial classique ne subsiste que dans les cas d'extrême respect.

L'âge classique a longuement expliqué que tout comme dans la conversation, le commerce épistolaire devait répondre aux exigences du savoir-vivre et de la bienséance. Pour ce qui concerne le style, celui-ci doit retrouver la grâce de la conversation, c'est-à-dire la négligence et le naturel :

Le style doit sentir la négligence et ne diffère guère du langage ordinaire. Les figures des orateurs n'y conviennent point non plus que les longues périodes. Il faut qu'il y paraisse quelque grâce, qui convie en toutes lettres à les lire et à les bien considérer, fuyant en telle sorte l'affectation que toutefois il n'y ait point de rusticité ni de mots ou façons de parler barbares ou impropres ${ }^{17}$.

Retrouver dans la lettre la conversation des honnêtes gens est la finalité revendiquée :

Il faut se faire une affaire sérieuse d'écrire une lettre : choisir bien son sujet et l'exposer avec toute la décence convenable. Les hauteurs et les bassesses sont également opposées à la bonne éducation et aux sentiments d'un cœur bien né. Le style doit être vif, net et concis, il faut éviter les lieux communs de la rhétorique, rien ne doit sentir l'affectation ${ }^{18}$.

Gellert insistera lui aussi sur le fait que ce n'est pas une conversation ordinaire et que tout n'est pas permis ce qui l'est à l'oral : "C'est une imitation d'une bonne conversation ${ }^{19}$."

Les secrétaires offraient de nombreux exemples de lettres adressées à toutes sortes de personnes et rédigées suivant leur rang et position sociale ou familière, et les modèles étaient multipliés à l'envi, ce que les traités de savoir-vivre du $\mathrm{XIX}^{\mathrm{e}}$ siècle continueront à augmenter encore dans des ouvrages servant de " code de la route " du commerce épistolaire (on a envie de dire de la mécanique du commerce épistolaire), tels ceux de la Comtesse de Bradi ${ }^{20}$, d'Henri Bescherelle ${ }^{21}$ ou d'Édouard Hocquart ${ }^{22}$, qui ont connu d'innombrables rééditions.

Les secrétaires avaient imposé des règles de style, des valeurs et des idéaux rhétoriques. Ainsi les secrétaires allemands des XVII ${ }^{\mathrm{e}}$ et XVIII ${ }^{\mathrm{e}}$ siècles conseillent d'éviter les mots étrangers, les expressions orales, les mots archaïques et démodés, comme les nouveaux qui viennent d'être mis à la mode. Il ne faut pas faire de trop longues phrases, éviter les répétitions et bannir l'emploi des parenthèses

17. Jean Puget de La Serre, Le Secrétaire à la mode ou Méthode facile d'écrire selon le temps, diverses lettres de compliments, amoureuses et morales..., Amsterdam, Elzevier, 1645 [1 ${ }^{\text {re }}$ éd. 1639], p. 45.

18. Eléazar de Mauvillon, Traité général du style avec un traité particulier du style épistolaire, Amsterdam, Mortier, 1751, p. 257.

19. Gellert, Briefe, nebst einer praktischen Abhandlung von dem guten Geschmacke in Briefen, Leipzig, Wendler, 1751, p. 3 : "freye Nachahmung des guten Gesprächs ».

20. Comtesse de Bradi, Le Secrétaire du dix-neuvième siècle faisant suite au savoir-vivre en France, Paris, Levrault, 1840.

21. L'Art de la correspondance. Nouveau manuel du style épistolaire, Paris, H. Dentu, 1865 [1 $1^{\text {re }}$ éd. 1838].

22. Secrétaire pour tout le monde, Paris, J. Langlumé et Peltier, 1845. 
qui alourdissent le texte de la lettre. La norme fondamentale est la clarté. Ce qu'il est important de signaler pour la civilité épistolaire en Allemagne est la prépondérance quasi absolue des modèles étrangers, bien entendu antiques, mais avant tout français qui sont la norme au XVII ${ }^{\mathrm{e}}$ siècle, à commencer par Hunold ou Bohse, célèbre à la fois par ses très nombreux écrits théoriques (en particulier en 1690 Der allzeitfertige Brieffsteller ${ }^{23}$ ) et ses romans qui témoignent de la manière dont on doit écrire des lettres. Le modèle français est prégnant et Bohse écrit d'ailleurs une traduction du Nouveau sécrétaire françois ou lettres schoisies sur toutes sortes de sujets de Charles Louis de Merville (Der neue Französiche Briefsteller) en 1730. Cela implique que l'on doit toujours adapter son style à la position sociale de la personne à laquelle on s'adresse, qu'il faut respecter la disposition conventionnelle et tout particulièrement quand on s'adresse à des personnes haut placées. Mais Bohse dénonce l'affectation, les allégories et les formules contraintes pour une plus grande pureté naturelle de la langue : "Une lettre ne doit pas être rédigée d'une autre manière que celle dont on parle quand on mène une conversation galante et à la cour. Tout ce qui est poétique et trop éloquent ne convient pas dans une lettre ${ }^{24}$. "Aussi, bien qu'il préconise la clarté dans la langue allemande, il s'autorise à admettre des mots français parce que le style français est "plus libre et plus coulant ". Bohse suit en cela Weise dans l'emploi et les tournures du style galant, qui n'hésite pas à l'introduction de termes français pour colorer cette politesse mondaine. Par exemple: Très estimé Amy - Conversation - passiren - votre très humble serviteur Affection - obligat - Qvaliteten - honêter Cavalier - galante Conduite - etc.

Le grand modèle est cette galanterie qui est un art de la distinction et une esthétique des douceurs, des belles manières, des hommages. Un art de vivre que le chevalier de Méré envisage en France comme une honnêteté brillante (" je crois que la bonne galanterie n'est autre chose que la parfaite honnêteté, accompagnée de vrais Agréments en tout ce qu'elle fait ou qu'elle dit, et d'une manière noble et délicate ${ }^{25}$ »). Pierre Ortigue de Vaumorière dans L'art de plaire dans la conversation, pense que l'air galant est l'exact équivalent de ce qu'était "l'urbanité des anciens Romains " avec ce je-ne-sais-quoi qui en caractérise la finesse civile.

Bohse affirme qu'il faut persuader son auditoire de ce qu'on lui expose et gagner dans le même temps son sentiment (,ihre Affekte also zu gewinnen ${ }^{26 “)}$. Il se sert du terme "Insinuationes » qui est en quelque sorte le sucre de ce commerce rendu agréable et persuasif par le style galant ${ }^{27}$. Les "Insinuationes " peuvent aller jusqu'à des flatteries pour conquérir la bienveillance du lecteur. Il donne

23. Éditions et rééditions de ses écrits sur l'épistolaire se comptent par dizaines.

24. August Bohse (Talander), Neu-Erleuterter Briefsteller, Leipzig, Gleditsch, 1697, p. 119 : „Ein Brief muß nicht anders abgefasset seyn als wie man in höflicher und galanter Conversation zu reden pfleget. Was poëtisch und allzu oratorisch klinget das schickt sich in keinen Brief."

25. Méré, Euvres complètes, Paris, Fernand Roches, 1930, p. 44-45.

26. Bohse, Neu-Erleuterter Briefsteller, p. 4.

27. Sur la galanterie en Allemagne, voir en particulier Jörn Steigerwald, Galanterie. Die Fabrikation einer natürlichen Ethik der höfischen Gesellschaft (1650-1710), Heidelberg, Universitätsverlag 
des exemples concrets à ces insinuations, emplois d'adjectifs agréables : "Les insinuations ne sont rien d'autre que des épithètes habiles et flatteuses ${ }^{28}$. " La finesse précieuse qui va de pair avec la clarté dans le style galant conquiert ainsi la bienveillance. Les exemples qu'il donne dans Des Galanten Frauenzimmers Secretariat-Kunst (1692) montre l'hésitation entre une forme galante et une certaine souplesse ${ }^{29}$.

Hunold (Menantes) suivra le modèle de Bohse en accentuant encore la prégnance du modèle français. Ainsi donne-t-il deux ans après son premier secrétaire Allerneusten Art Höflich und Galant zu Schreiben (Hamburg, 1702) un volume bilingue allemand-français, Lettres choisies des meilleurs et plus nouveaux Auteurs françois (Hamburg, 1704), pour que "même ceux qui ne comprennent pas le français puisse prendre connaissance d'après la traduction des belles et nobles expressions des épistoliers français ${ }^{30}$ ". Ceux-ci sont entre autres tout particulièrement Richelet, Voiture, Balzac, Méré, d'Her, Bussi, Milleran, Richelieu. Il revendique dans sa préface („Von dem Stylo im Schreiben“) cependant un certain naturel particulièrement dans les lettres d'amour („Die Natur ist uns allhier weit nöthiger als die Kunst“). C'est la raison pour laquelle il ne faut pas attendre de l'ordre dans les lettres d'amour.

Benjamin Neukirch (Anweisung zu Teutschen Briefen, 1709) a une préférence pour le style galant qui assure le charme, le tour, le decorum qui lui semble plus naturel et plus souple. Et, pour Gellert, le style galant doit avoir ce naturel qui caractérisait Cicéron. Cela sera repris par Stockhausen : "Chez [Cicéron], rien ne semble artificiel ou recherché ; tout donne l'impression qu'il aurait pu parler comme il parle ${ }^{31}$ ". Mais ce naturel reste en un premier temps celui de la sprezzatura castiglionesque, une seconde nature résultant d'un apprentissage et d'une culture, une forme urbaine, cultivée, propre aux humanistes et aux hommes de cour.

Les choses changent vers le milieu du siècle, lorsqu'on se tourne vers d'autres modèles : le modèle anglais fait déjà son apparition avec Aldinor, traducteur et éditeur des Choice Letters - Auserlesener Briefe, Aus einigen Von den galantesten und berühmtesten Englischen Autoribus gesammelt (Hannover, 1725). Avec la publication de Paméla (1740), la lettre pénètre le roman et devient aussi un autre modèle d'expression naturelle. "Si l'on déclare que Paméla, Clarissa, Grandison, sont de bons romans, on doit sans nul doute estimer aussi que ce sont de belles lettres " ("Wenn die Pamela, Clarissa und Grandison für gute Romane sind

Winter, 2011, et Ruth Florack, Rüdiger Singer (dir.), Die Kunst der Galanterie. Facetten eines Verhaltensmodells in der Literatur der frühen Neuzeit, Berlin/Boston, de Gruyter, 2012.

28. Méré, Neu-Erleuterter Briefsteller, p. 218 : „Die Insinuationes seynd nichts anders als geschickte und schmeichelnde Beywörter.“

29. On trouvera des analyses précises de ces lettres dans l'ouvrage de Reinhard M.G. Nickisch, Die Stilprinzipien in den deutschen Briefstellern des 17. und 18. Jahrhunderts, Göttingen, Vandenhoeck \& Ruprecht, 1969.

30. Préface (sans pagination).

31. Stockhausen, Grundsätze wohleingerichteter Briefe, p. 38 : „Nichts scheinet an ihm gekünstelt oder gesucht ; alles hat das Ansehen, als ob er so hätte reden müssen, wie er redet.“ 
erkläret worden, so muß man sie gewiß auch für schöne Briefe halten ") écrit-il dans un ajout à l'édition de 1756 (la troisième), car l'époque des romans par lettres est arrivée.

L'originalité de la démarche de Richardson fut de trouver dans le modèle du secrétaire et du recueil de lettres un ferment pour la création. Plus directe que l'essai, la lettre permet l'exposition d'une morale domestique dans la tradition du Spectateur, tout en contenant en germe le romanesque. Ainsi les Lettres familières de Richardson renferment-elles, outre un code sentimental, domestique et moral, l'esquisse de petits romans d'analyse où les élans du cœur s'affrontent aux principes et où différents types de problème de conduite sont évoqués. Elles préfigurent le grand roman épistolaire qu'est Paméla ou La vertu récompensée 32 (1740).

Communication et épanchement. Tout solitaire est coupable et la lettre est un aveu ("Votre misérable Paméla ", "Oh ! ayez pitié de votre malheureuse fille "), une confession des mouvements de la sensibilité. Aveu aussi en tant qu'elle permet d'extérioriser par l'écriture les fantômes de l'âme. Rilke parlait ainsi d'autotraitement ("Selbstbehandlung "). Catharsis manifeste : vider son cœur, s'épancher, trouver l'exutoire. La lettre, miroir et révélateur des états secrets de l'âme, a affaire avec le secret.

L'écriture est certes pour Paméla un exercice de la vertu, mais la prolixité épistolaire tend à l'autoconsommation et à l'autoconsumation: la vertu se nourrit de la vertu comme le papier appelle le papier et la fiction, la fiction. Paméla devient un être de papier : "J'ai cousu autour de mes hanches dans ma jupe de dessous, où j'espère qu'ils sont en sûreté, les papiers qui contiennent le détail de mon peu de réussite et de ce qui s'ensuit ${ }^{34}$ ". Ces papiers, partie figée d'elle-même, lui collent à la peau, et ses protestations de vertu sont évidemment une barrière qui l'empêche de se jeter à cœur et corps perdus dans l'amour. Mais ces papiers cachés dans les lieux les plus secrets appellent le lecteur, invitent à leur lecture et on ne saurait trop rappeler que le geste épistolaire semble faire effort pour surmonter une solitude première dont le dialogue épistolaire porte les traces.

En France, mais également en Allemagne, on rejettera progressivement les formes du cérémonial pour un commerce plus souple. Louis Philipon de la Madelaine, dans le chapitre "Cérémonial observé dans les lettres " de ses

32. Le titre complet mérite d'être cité intégralement: Suite de lettres familières, écrites par une belle jeune personne à ses parents, et publiées afin de cultiver les principes de la vertu et de la religion dans les esprits des jeunes gens des deux sexes; ouvrage qui a un fondement vrai et qui, en même temps qu'il entretient agréablement l'esprit par une variété d'incidents curieux et touchants, est entièrement purgé de toutes ces images qui, dans trop d'écrits composés pour le simple amusement, tendent à enflammer le cœur au lieu de l'instruire.

33. Rilke dans une lettre à Lou Andreas-Salomé du 20 janvier 1912 (Rainer Maria Rilke, Lou AndreasSalomé, Briefwechsel, Wiesbaden, Insel, 1952, t. 1, p. 385).

34. Paméla ou la vertu récompensée, traduction par l’Abbé Prévost, Amsterdam et Paris (s. éd.), 1784, p. 491. 
Modèles de lettres sur différents sujets ${ }^{35}$ (1761), souligne qu'il faut toujours respecter les convenances en usage, mais que l'humble et obéissante servitude " n'est plus que de l'extrême civilité ». Il déplore ce que l'Encyclopédie appelle " les vains compliments d'étiquette, restes de barbarisme, fruits de la servitude et de la décadence du goût " (article "Lettre "). Karl Philipp Moritz, dans Anleitung zum Briefschreiben (Berlin, 1783) par exemple, s'indigne comme nombre de ses contemporains contre la soumission aveugle inculquée à la jeunesse dans les secrétaires avec l'emploi des titres et toute la pompe du cérémonial. Il oppose à cela une expression simple du respect. Le XVIII ${ }^{\mathrm{e}}$ siècle éprouve généralement une grande antipathie envers les formules de politesse formelles et exagérées qui suscitent le dégoût en raison de leur hypocrisie, leurs feintes caresses, leurs flatteries mensongères et leurs soumissions exagérées et indignes, au point même que l'on en vient à rejeter secrétaires et manuels. "Quiconque raisonne, met en ordre, pense avec vivacité, pourra rédiger à sa guise ${ }^{36}$ " ainsi que l'écrit Stockhausen. Autrement dit, si l'on sait penser et s'exprimer, on peut négliger toutes les règles. On peut dire qu'à la fin du XVIII ${ }^{\mathrm{e}}$ siècle la notion d'individualité et de subjectivité ${ }^{37}$ prend le dessus en s'affranchissant de l'imitation des modèles. Ainsi Moritz :

Ein Briefsteller sey noch so gut, so stiftet er allemal mehr Schaden als Nutzen. Denn auch gute Briefe, wenn sie nachgeahmt werden, verlieren in der Nachahmung ihren ganzen Werth : weil der Werth eines Briefes darin bestehe, daß er ein getreuer Abdruck von der eignen Wendung in den Gedanken, und in dem mündlichen Ausdruck eines jeden sey. ${ }^{38}$

Une nouvelle ère commence et les préoccupations qui se font jour sont autres que ces règles de civilité qui, si elles subsistent bien évidemment, ne sont plus qu'un code attendu et non plus un art, comme ce fut le cas à l'époque de la galanterie. Désormais la revendication du naturel est de plus en plus profonde, comme en témoigne en 1748 Der Gesellige, topos que l'on retrouvera par la suite chez Knigge dans Ueber den Umgang mit Menschen (1788) :

Dieses sind Briefe [die freundschaftlichen Briefe], zu derer Verfertigung noch kein Menantes einen Briefsteller geschrieben und Regeln gegeben hat. Das Herz, die Natur, die Empfindungen sagen sie in die Feder, und sie gerathen allemal schön. Sie sind nicht mit Fleiß witzig gemacht, und sind von sich selbst voller Witz. Der Witz der freundschafltichen Briefe gleichet einen schönen Frauenzimmer, das weder Schminke noch prächtige Kleider, noch eine angenommene Stellung beobachtet, sich zu erheben, sondern von selbst und ungesuchet, auch in nachlässiger Kleidung am meisten, reizet : ihr natürlicher Wuchs fällt nur desto schöner in die Augen, je weniger Putz bey ihr angetroffen wird. Sie gefällt allemal, ohne daß sie daran gedenket, ohne daß sie zu gefallen sucht. Die Munterkeit, die vertrauliche Ungezwungenheit, die zuversichtliche Dreistigkeit, der Scherz, und die Verbannung grosser Titel und der Ceremonien, bringen ein Leben in die Schreibart, welches die Mutter des wahren Witzes ist. [...] Solche Briefe sind es, die die Abwesenheit versüssen. ${ }^{39}$

35. Bouillon, Jean Brasseur, 1761, p. 41-52.

36. Stockhausen, Grundsätze wohleingerichteter Briefe, p. 158 : „Wer vernünftig, ordentlich und lebhaft denket, der kann schreiben wie er will.“

37. Nul doute que la mode de l'amitié n'ait joué son rôle dans cette évolution qui préfère l'épanchement spontané des âmes aux règles établies.

38. Karl Philipp Moritz, Anleitung zum Briefschreiben, Berlin, Wever, 1783, p. VIII.

39. Der Gesellige, t. I \& II, nº 72, 1748, p. 588-590. 
Les fictions, les romans épistolaires, à commencer par La Religieuse portugaise, les romans de Richardson et toutes les littératures romanesques du XVIII ${ }^{\text {e }}$ siècle écrites sous forme de lettres, ont ébranlé la mode d'un style galant ou convenu pour revendiquer un langage plus naturel issu de l'intimité d'un sujet. Le dialogue à distance a également révélé la solitude de l'épistolier renvoyé à ses propres fantasmes et fantômes, la lettre se réfléchissant alors elle-même tout en questionnant sa légitimité et son effıcacité pragmatique.

L'épistolaire retrouve aussi le dilemme de tout savoir-vivre entre politesse et hypocrisie, spontanéité comme marque d'authenticité et l'artifice et sa feinte, sa fiction.

La lettre est un genre insaisissable dans le filet des règles qui la régissent, mais elle est aussi fragmentée et sans fin parce que toujours à recommencer en raison d'une mélancolie fondamentale qui porte le deuil de la voix, deuil de la présence de l'autre, du temps qui s'échappe :

Les lettres ne sont que tourment ; elles viennent d'un tourment incurable, elles ne peuvent produire qu'incurable tourment... La grande facilité d'écrire des lettres doit avoir produit dans le monde, d'un point de vue purement théorique, une terrible dislocation des âmes ; c'est un commerce avec des fantômes, non seulement avec celui du destinataire, mais encore avec le sien propre ${ }^{40}$.

Kafka pose ainsi le problème même de la communication épistolaire. Tout comme la Religieuse portugaise qui insiste sur la valeur pragmatique profonde de la lettre : "Il me semble que je vous parle, quand je vous écris, et que vous m'êtes un peu plus présent ${ }^{41}$ ", Kafka souligne la confusion de ces représentations : celui à qui l'on s'adresse est une représentation, un fantôme. S'il crée tel prolifique échange épistolaire avec Felice, c'est que celle-ci est insignifiante ${ }^{42}$ et donc propre à l'investissement et dans le même temps - apparent paradoxe - à la tenir infiniment à distance, à en faire un personnage de lettres, un correspondant imaginaire loin de toute réalité tangible. "Ce qui me gênait, outre d'autres scrupules d'ailleurs, c'était positivement la peur de voir dans sa réalité la femme qui m'écrivait ${ }^{43}$ " et l'idée de la voir lui semble signer la fin de l'échange : se fiancer signifie pour lui devenir un épistolier peu prolixe. Et lucidement, dans la même lettre, il confesse qu' " une grande correspondance est le signe que quelque chose ne va pas $^{44}$ ". D'ailleurs, certaines lettres sont-elles vraiment adressées à Felice ou ne sont-elles pas plutôt écrites contre ses parents ? Vincent Kaufmann

40. Franz Kafka, Lettres à Milena, Paris, Gallimard, 1956, p. 260.

41. Cité par Richelet, Les plus belles lettres françoises sur toutes sortes de sujets, La Haye, de Voys, t. 1,1708 , p. 163.

42. "Visage osseux et insignifiant, qui portait franchement son insignifiance " (Knochiges, leeres Gesicht, das seine Leere offen trug“), Tagebuch, 20. August 1912, Frankfurt a.M., Fischer, 1994. Sur ce journal, cf. Florence Bancaud, Le Journal de Kafka ou l'écriture en procès, Paris, CNRS, 2001.

43. Lettre à Max Brod à propos d'un séjour à Marienbad avec Felice en juillet 1916 (cité par Vincent Kaufmann, L’Équivoque épistolaire, Paris, Les Éditions de Minuit, 1990, p. 21).

44. Lettre à Felice du 15 août 1913 (Frankfurt a.M., Fischer, 2015, nº 305) : „Ein großer Briefverkehr ist ein Zeichen für, daß etwas nicht in Ordnung ist. Der Frieden braucht keine Briefe.“ 
a bien montré que pour Kafka, écrire sert à mettre en échec l'Autre, " pour faire la démonstration que l'Autre, ça ne marche pas, que ça n'existe pas, et qu'aucune médiation, aucun tiers ne tient le coup ${ }^{45}$ " et que l'épistolaire fonctionne comme ligne de fuite par rapport au symbolique. S'il évoque une maladresse de Kafka, c'est qu'il y a mal-adresse, l'adresse impossible que Kafka évoque par une image forte dans une lettre à Milena : "les baisers écrits ne parviennent pas à destination, les fantômes les boivent en route ${ }^{46}$ ". On comprend dès lors que la lettre la plus authentique est celle qu'on n'envoie pas. Ainsi de la Lettre au père, long autoportrait du fils malheureux adressant son tourment accusateur à la figure paternelle, lettre qu'il ne donnera jamais à lire au père - ni d'ailleurs à Milena à qui Kafka le promettait, donc à personne, lettre destinée à ne pas être lue.

45. Kaufmann, L'Équivoque épistolaire, p. 72.

46. Kafka, Lettres à Milena, p. 260. 